\title{
T-wave inversion in the aVL lead is associated with severity of stenosis in the LAD artery
}

\author{
Mahsa Behnemoon ${ }^{\star}$, Hamid Sibir
}

Received 05 June 2020, Accepted for publication 04 October 2020

\begin{abstract}
Background \& Aims: The clinical value of T wave inversion (TWI) in the lead aVL in diagnosing coronary artery disease (CAD) remains unclear. Hence, we aim to evaluate the association between TWI in the aVL lead and the severity and location of coronary artery stenosis in elective coronary angiography.

Material and Method: Electrocardiograms (ECGs) of 167 consecutive patients undergoing elective coronary angiography were analyzed. All patients had chronic stable angina. Patients with secondary T wave inversion had been excluded (67 patients). Detailed ECG and coronary angiographic findings were interpreted by experienced cardiologists. A total of 50 patients with isolated T wave inversion in the aVL lead (without other ECG abnormalities) and 50 control subjects with normal electrocardiogram were studied.

Results: Of the 100 enrolled subjects, 54 were males and 47 were females and the mean age in this study was $63.77 \pm 9.2$ years old (range 44-81). There was a significant relationship between the severity of stenosis in the LAD artery and TWI in the aVL lead $(\mathrm{P}<0.0001)$. The sensitivity and specificity of TWI in the aVL lead for LAD artery stenosis were $74 \%$ and $65 \%$, respectively.

Conclusion: According to our study, TWI in the aVL lead exhibits a meaningful correlation with LAD artery stenosis, which is mostly associated with the stenosis of the middle portion of the artery, with relatively high sensitivity and specificity.
\end{abstract}

Keywords: Coronary artery diseases, ECG, T wave inversion, aVL lead

Address: Urmia University of medical science, Urmia, Iran

Tel: +984432234897

Email: mm_bk_80@yahoo.com

\section{Introduction}

Resulting from atherosclerotic plaque formation within the internal lumen (intima) of the coronary arteries, Coronary artery disease (CAD) presents itself with manifestations heralding decreased blood flow such as acute coronary syndrome or chronic stable angina (1). Throughout the globe, $\mathrm{CAD}$ is recognized as a prevalent cause of death, resulting in an array of complications which impose a considerable economic burden to the countries (2). In Iran the high CAD associated prevalence and morbidity, is a major health concern (3). Of note, while similar to some Middle East countries, the prevalence of coronary risk factors and

\footnotetext{
${ }^{1}$ Assistant professor of cardiology, Urmia university of medical science, Urmia, Iran (Corresponding author)

${ }^{2}$ Medical doctor, Urmia university of medical science, Urmia, Iran
} 
metabolic syndrome in Iran is higher compared to most Western countries (4)

An electrocardiogram (ECG) is a non-invasive bedside diagnostic tool with a well- established role in the diagnosis of CAD and in particular acute coronary syndrome (ACS) (5). Bodies of evidence imply that obtaining an ECG at admission provides important prognostic information in most patients; ST-T changes in ECG reflect myocardial ischemia or myocardial necrosis after myocardial injury (6-10). The prognostic value of the aVL lead in patients with angina pectoris remains vague. However it appears that alterations in this lead could be a sensitive sign for Acute MI, even prior to the occurrence of ST elevation (11-13). T wave inversion (TWI) in the aVL lead, as a reciprocal change following infarction of the inferior cardiac wall, has been observed in lesions of the right coronary artery (RCA); However, recent studies hint at the association of TWI with significant lesions of the left anterior descending artery (LAD), describing TWI as a manifestation of acute MI (11, 13-17). In fact, the aVL lead can foretell reciprocal changes of the inferior wall sooner than the occurrence of STE in inferior leads in instances of MI. Obtaining serial ECGs will assist in better understanding such dynamic changes following MI. $\mathrm{T}$ wave inversion in the aVL lead resulting from chronic LAD lesions, will remain the same throughout serial obtained ECGs; While, reciprocal changes of inferior wall MI should evolve from TWI to ST depression in reciprocal leads, followed by ST elevation in leads II, III and avf $(11,17)$. However, knowledge pertaining to the prognostic significance of the aVL lead as a predictor for CAD in patients with chronic stable angina is sparse and inconclusive. Hence, we aim to evaluate the association between TWI in the aVL lead and the severity and location of coronary artery stenosis in elective coronary angiography.

\section{Methods}

\section{Study populations:}

This prospective observational study was conducted during 6 months, from June to November 2016, and enrolled 167 patients referred to Talegani Hospital for invasive coronary angiography with a history of stable $\mathrm{CAD}$ as proved by non-invasive methods (ECG, echocardiography, treadmill exercise, myocardial perfusion imaging or multi-slice computed tomography coronary angiography). The exclusion criteria included clinical situations that may result in secondary aVL lead $\mathrm{T}$ wave inversion and/or ST segment depression secondary to bundle branch block either left (LBBB) or right $(\mathrm{RBBB})$, paced ventricular rhythms, LV strain pattern due to chronic hypertension or systolic heart failure. Informed consent was obtained from all patients, and the study was approved by the ethics committee of Urmia University of medical sciences.

\section{Electrocardiogram:}

ECG was recorded in the 12-lead format at a paper speed of $25 \mathrm{~mm} / \mathrm{s}$ and fully interpreted with more focus on $\mathrm{T}$ wave morphology in the aVL lead. Assessments were made by two independent expert cardiologists. Based on a joint recommendation of the American Heart Association (AHA), the American College of Cardiology Foundation (ACCP) and the Heart Rhythm Society (HRP) (18), T wave inversion was defined as T wave $\leq-0.1 \mathrm{mV}$, compared with the baseline from the end of the $\mathrm{T}$ wave to the beginning of the $\mathrm{P}$ wave.

\section{Coronary angiography:}

Coronary angiograms were analyzed by interventional cardiologists. Left anterior descending (LAD), left circumflex (LCX), and right coronary artery (RCA) and all their respective branches were defined and plotted on the data sheet. The definition of the coronary tree segments is based on the SYNTAX score classification (19). Definitions are as follows: Proximal LAD: proximal to, and including, the first major septal branch; mid LAD: from the immediate distal to the origin of first septal branch, extending to the point where 
LAD forms an angle (in instances which this angle is not identifiable, this segment ends at one half of the distance from the first septal branch to the apex of the heart); distal LAD segment: the terminal portion of LAD, beginning at the end of previous segment and extending to or beyond the apex; Proximal LCX artery; the main stem of the circumflex artery from its origin of left main artery, also including the origin of first obtuse marginal branch; Distal circumflex artery: from the stem of the circumflex distal to the origin of the most distal obtuse marginal branch, continuing along the posterior left atrioventricular groove; Proximal RCA: From the ostium to one half of the distance to the acute margin of the heart; Mid RCA: from the end of first segment to the acute margin of heart; distal RCA: from the acute margin of the heart to the origin of the posterior descending artery.

\section{Statistical analysis:}

Descriptive statistics were used to describe the data. For categorical variables, frequencies and percentages were reported. Differences between groups were analyzed using Pearson's chi squared test. For continuous variables, means and standard deviations $( \pm \mathrm{SD})$ were presented by using SPSS version 16.0 (SPSS Inc., Chicago, IL, USA). P < 0.05 was considered as significant.

\section{Results}

Out of the 167 patients enrolled in this study, 34 patients were excluded due to meeting the exclusion criteria (LBBB: 17 patients; RBBB: 10 patients; paced ventricular rhythm: 2 patients; LV strain pattern due to chronic hypertension or systolic heart failure: 5 patients). An additional 34 patients were also excluded due to non-specific changed in $\mathrm{T}$ wave morphology in leads other than aVL. In conclusion, a total of 50 patients with isolated $\mathrm{T}$ wave inversion in the aVL lead and 50 control subjects with normal electrocardiogram were studied. Of the 100 enrolled subjects, 54 were males and 47 were females and the mean age in this study was 63.77 \pm 9.2 years old (range 44-81).

Among 50 patients with T wave inversion (TWI) in the aVL lead, 6 (12\%), 23(46\%) and 21 (42\%) patients showed stenosis of $<50 \%, 50-70 \%$ and $>70 \%$ in the LAD artery, respectively. There was also a significant relationship between the severity of stenosis in the LAD artery and the TWI in the aVL lead $(\mathrm{P}<0.0001)$. The correlation between the severity of stenosis in the RCA and LCX arteries with TWI in the aVL lead was not significant $(\mathrm{P}=0.436, \mathrm{P}=0.08$ respectively) (Table 1).The sensitivity and specificity of TWI in the aVL lead for LAD artery stenosis were $88 \%$ and $65 \%$, respectively.

In this study, 11 (26\%), 39 (78\%) patients had a lesion in the proximal and mid-segment of LAD respectively. Although TWI was seen in the midsegment of LAD of most cases, a significant relationship between the location of a stenosis in the LAD, RCA and LCX arteries with the TWI in the aVL lead was not observed $(\mathrm{P}=0.075, \mathrm{P}=0.085, \mathrm{P}=0.0156$, respectively) (Table 2). $\mathrm{T}$ wave inversion in the aVL lead demonstrated a sensitivity of $74 \%$ and specificity of $65 \%$ for predicting mid-segment LAD lesions.

\section{Discussion}

In this study, the correlation between TWI in the aVL lead and the severity of LAD artery stenosis was assessed. This correlation was statistically significant in the group with inverted $\mathrm{T}$ waves as compared to the control group and the severity of the stenosis was also related to the inversion of the $\mathrm{T}$ wave $(\mathrm{P}<0.0001)$. In our study, TWI in the aVL lead had a sensitivity of $74 \%$ and specificity of $65 \%$, for the prediction of the severity of LAD stenosis.

In a study by Hatem L. Farhan et al. conducted in 2010 with the aim of evaluating the inverse T-diagnostic 
value of aVL in the diagnosis of coronary artery disease, ECGs and angiographic evaluations of 257 patients considered the TWI in the lead avl as the only variable that significantly predicts the lesion in the middle segment of the LAD artery $(\mathrm{OR}=2.93, \mathrm{P}=0.001)(13)$. Also, TWI in the aVL lead predicted a lesion in the LAD artery with a sensitivity of $61 \%$, which was consistant with our findings (Sensitivity of $74 \%, \mathrm{P}<0.0001$ ). In our study, in terms of location, lesions of the median segment were more prevalent $(74 \%)$ and had a sensitivity of $74 \%$ with $\mathrm{OR}=5.5$. A possible cause of variation between findings can be attributed to difference in sample sizes. However, both studies have focused on the predictive value of TWI for LAD artery stenosis.

In a study by Getaw Worku Hassen et al. performed in 2014 (16), with the aim of assessing the specificity of TWI in the aVL lead as an indicator of LAD artery lesions, 125 ECGs and angiography data with mid-LAD lesions were studied. One hundred and six patients $(84.8 \%)$ had a lesion $>50 \%$ and 19 patients $(15.2 \%)$ had a lesion $<50 \%$. Isolated TWI in lead aVL had an overall sensitivity of $76.7 \%$, a specificity of $71.4 \%$, a positive predictive value of $92 \%$ and a negative predictive value of $41.7 \%$. Despite differences in methodology with our study, the sensitivity and specificity of the TWI for midLAD lesion in our study was in line with the aforementioned study, indicating a significant relationship between TWI and LAD artery stenosis, especially the segmental mid portion stenosis of the artery. In another study, it reported that $\mathrm{T}$ wave inversion in the aVL lead, regardless of other $\mathrm{T}$ wave changes had a sensitivity of $32.9 \%$ and specificity of $48.2 \%$ for predicting mid-LAD lesions, which is in disagreement with our findings, showing that $\mathrm{T}$ wave inversion in lead aVL regardless of other $\mathrm{T}$ wave changes had low diagnostic values for predicting midLAD lesions (20). In general, both studies have focused on the predictive value of TWI for LAD artery stenosis.
Our limitations were relatively small sample size and short time interval of the study, so we recommend other large scale case control studies with considering of other conflicting factors (e.g. coronary risk factors, age, sex etc.) in order to evaluate the precise role of the aVl lead in detection of the probable coronary artery stenosis in the near future.

\section{Conclusion}

According to our study, TWI in the aVL lead exhibits a meaningful correlation with LAD artery stenosis, which is mostly associated with the stenosis of the middle portion of the artery, with relatively high sensitivity and specificity. In conclusion, we report that TWI in the aVL lead in patients with angina pectoris, CAD is likely to be related to mid segment LAD stenosis. In cases which further and more extensive CAD symptoms are present, the patient is better to be referred to a cardiologist for consult and treatment.

\section{References}

1. Williams RA. Cardiovascular disease in African American women: a health care disparities issue. J Natl Med Assoc 2009;101(6):536-40.

2. Fiuza M. Metabolic syndrome and coronary artery disease. Rev Port Cardiol 2012;31(12):779-82.

3. Nozari Y, Hashemlu A, Hatmi ZN, Sheikhvatan M, Iravani A, Bazdar A, et al. Outcome of coronary artery bypass grafting in patients without major risk factors and patients with at least one major risk factor for coronary artery disease. Indian J Med Sci 2007;61(10):547-54.

4. Ebrahimi M, Kazemi-Bajestani S, Ghayour-Mobarhan M, Ferns G. Coronary artery disease and its risk factors status in Iran: a review, Iran Red Crescent Med J 2011;13(9):610-23.

5. Zimetbaum PJ, Josephson Mark E. Use of the electrocardiogram in acute myocardial infarction. N Engl J Med 2003;348(10):933-40. 
6. Cannon CP, McCabe CH, Stone PH, Rogers WJ, Schactman M, Thompson BW, et al. The electrocardiogram predicts one-year outcome of patients with unstable angina and non-Q wave myocardial infarction: results of the TIMI III.Registry ECG Ancillary Study. J Am Coll Cardiol 1997;30(1):133-40.

7. Nyman I, Areskog M, ARESKOG NH, Swahn E,Wallentin L. Very early risk stratification by electrocardiogram at rest in men with suspected unstable coronary heart disease. J Intern Med 1993;234(3):293-301.

8. Okada M, Yotsukura M, Shimada T, Ishikawa K. Clinical implications of isolated $\mathrm{T}$ wave inversion in adults: Electrocardiographic differentiation of the underlying cause of this phenomenon. Am Heart J 1994;24(3):73945.

9. Rhinehardt J, Brady WJ, Perron AD, Mattu A. Electrocardiographic manifestations of Wellens' syndrome. Am J Emerg Med 2002;20(7):638-43.

10. Gurm H S, Topol E J. The ECG in acute coronary syndromes: new tricks from an old dog. Heart 2005;91(7):851-853.

11. Birnbaum Y, Sclarovsky S, Mager A, Strasberg B, Rechavia E. ST segment depression in aVL: a sensitive marker for acute inferior myocardial infarction. Eur Heart J 1993;14(1):4-7.

12. Hira RS, Wilson JM, Birnbaum Y. Introducing a new algorithm in inferior ST-segment elevation myocardial infarction to predict the culprit artery and distinguish proximal versus distal lesions. Coron Artery Dis 2011;22(3):165-70.

13. Farhan HL, Hassan KS, Al-Belushi A, Sallam M, AlZakwani I. Diagnostic value of electrocardiographic T wave inversion in lead aVL in diagnosing coronary artery disease in patients with chronic stable angina. Oman Med J 2010;25(2):124.

14. Kracoff OH, Adelman AG, Marquis J-F, Caspi A, Aldridge HE, Schwartz L. Twelve-lead electrocardiogram recording during percutaneous transluminal coronary angioplasty: Analysis of reciprocal changes. J Electrocardiol 1990;23(3):191-8.

15. Kracoff $\mathrm{OH}$, Adelman AG, Oettinger M, Ayzenberg O, Epstein M, Margulis G, et al. Reciprocal changes as the presenting electrocardiographic manifestation of acute myocardial ischemia. Am J Cardiol 1993;71(15):135962.

16. Hassen GW, Costea A, Smith T, Carrazco C, Hussein H, Soroori-Rad B, et al. The neglected lead on electrocardiogram: $\mathrm{T}$ wave inversion in lead aVL, nonspecific finding or a sign for left anterior descending artery lesion? J Emerg Med 2014;46(2):165-70.

17. Hassen GW, Talebi S, Fernaine G, Kalantari H. Lead aVL on electrocardiogram: emerging as important lead in early diagnosis of myocardial infarction? Am J Emerg Med 2014;32(7):785-8.

18. Rautaharju PM, Surawicz B, Gettes L. AHA/ACCF/HRS recommendations for the standardization and interpretation of the electrocardiogram: part IV: the ST segment, $\mathrm{T}$ and $\mathrm{U}$ waves, and the QT interval a scientific statement from the American Heart Association Electrocardiography and Arrhythmias Committee, Council on Clinical Cardiology; the American College of Cardiology Foundation; and the Heart Rhythm Society: Endorsed by the International Society for Computerized Electrocardiology. Circulation 2009;119:e241-e250.

19. Zipes DP, Libby P, Bonow RO, Mann DL, Tomaselli GF. Braunwald's Heart Disease E-Book: A Textbook of.Cardiovascular Medicine: Elsevier Health Sciences; 2018.

20. Nakanishi N, Goto T, Ikeda T, Kasai AJBo. Does T wave inversion in lead aVL predict mid-segment left anterior descending lesions in acute coronary syndrome? A retrospective study. BMJ open 2016;6(2):e010268. 